\title{
Computed Tomography of the Chest and Abdomen without Contrast
}

National Cancer Institute

\section{Source}

National Cancer Institute. Computed Tomography of the Chest and Abdomen without

Contrast. NCI Thesaurus. Code C137899.

Computed tomography of the chest and abdomen without the use of a contrast agent. 